\title{
Cosmological constant and time delay
}

\author{
T. Schücker ${ }^{1}$ and N. Zaimen ${ }^{2}$ \\ ${ }^{1}$ Centre de Physique Théorique, CNRS-Luminy, Case 907, 13288 Marseille Cedex 9, France \\ Unité Mixte de Recherche (UMR 6207) du CNRS et des Universités Aix-Marseille 1 et 2 et Sud Toulon-Var, \\ Laboratoire affilié à la FRUMAM (FR 2291); also at Université de Provence, France \\ e-mail: thomas.schucker@gmail.com \\ ${ }^{2}$ Laboratoire de Physique Théorique, Université Mentouri, 25000 Constantine; also at Université M'Hamed Bouguerra, \\ 35000 Boumerdes, Algeria \\ e-mail: zaimennoureddine@yahoo.fr
}

Received 24 January 2008 / Accepted 22 March 2008

\section{ABSTRACT}

\begin{abstract}
Aims. The effect of the cosmological constant on the time delay caused by an isolated spherical mass is calculated. Methods. We integrate the geodesic equations without using the lens equation.

Results. We compare our results to a recent observational bound on the time delay of the lensed quasar SDSS J1004+4112.
\end{abstract}

Key words. cosmology: theory

\section{Introduction}

Time delay is one of the four classical tests of general relativity. The first experimental confirmation was made in 1968 by Shapiro, who measured a time delay of $240 \mu$ s for a (one-way) travel-time of 10 min between Mercury and Earth. Last October Fohlmeister et al. (2007) published a lower bound of 5.7 years to a time delay of cosmological nature: the travel-time between the quasar SDSS J1004+4112 and Earth is roughly $10^{10}$ years. The lens was known to be a cluster of approximate mass $5 \times 10^{13}$ solar masses. One month earlier Rindler \& Ishak (2007) had corrected the wide held believe that the cosmological constant does not change the deflection angles of light.

While there has never been any debate in the literature about whether the time delay does depend on the cosmological constant, the controversy about whether the deflection angles depend on the cosmological constant was resolved within four months after publication of Rindler \& Ishak's work. The authors of two previous papers that supported the independence of the deflection angle with respect to the cosmological constant Lake (2007) and Sereno (2007) confirmed the dependence. In his first version of november, Sereno however finds a dependence different from Rindler \& Ishak's. In December, Schücker (2007) published an independent calculation confirming Rindler \& Ishak's result. In January Sereno (2007) published a second version of his work now agreeing with Rindler \& Ishak (2007). In this work Sereno also derived a formula for the time delay with cosmological constant. Earlier calculations of the time of flight were published by Kerr et al. (2003) and Kagramanova et al. (2006). We note the analysis of Bakola et al. (2007) that studies extreme lensing by black holes including a positive cosmological constant. In this paper, we compute the time delay between the most closely-aligned images of the quasar (C and D) as a function of the cosmological constant. We assume that the cluster is static and spherically symmetric and that its mass dominates all other lensing masses in the universe. The fact that there are five images of the quasar does indicate that the cluster is not perfectly spherical.

\section{The method}

We consider a universe, that is empty apart from one static, spherical, non-rotating mass $M$, the lens $L$. A source $S$, at rest with respect to the lens, emits photons, which are observed at nostra terra $T$, which is also assumed to be at rest. We neglect the masses of the source and Local Group. We use polar coordinates $(r, \theta, \varphi)$ centered on the lens. Because of spherical symmetry, the photons' trajectory is in a plane that we take to be $\theta=\pi / 2$. The angle $\varphi$ is measured with respect to the axis defined by the source, $\varphi_{\mathrm{S}}=0$. With a cosmological constant, the gravitational field outside the mass $M$ is given by the Kottler metric,

$$
\begin{aligned}
\mathrm{d} \tau^{2} & =B \mathrm{~d} t^{2}-B^{-1} \mathrm{~d} r^{2}-r^{2} \mathrm{~d} \varphi^{2}, \\
\theta & =\pi / 2, \quad B=1-\frac{2 G M}{r}-\frac{1}{3} \Lambda r^{2} .
\end{aligned}
$$

The source has polar coordinates $\left(r_{\mathrm{S}}, 0\right)$, the Earth is at $\left(r_{\mathrm{T}}, \varphi_{\mathrm{T}}\right)$. We define $\alpha$ and $\alpha^{\prime}$ to be the two physically measured angles between the images and the cluster center and denote by $r_{0}$ and $r_{0}^{\prime}$ the peri-lenses of the two light rays. We write $t_{\mathrm{S}}$ and $t_{\mathrm{T}}$ for the coordinate times of flight from the source to the peri-lens and from the peri-lens to Earth, see Fig. 1.

We assume that $\Lambda r^{2} / 3<9 / 10$ in order to avoid the coordinate singularity at the equator of the de Sitter sphere. We also assume that $\delta:=G M / r_{0} \ll 1, \alpha \ll 1$ and likewise for their primed quantities, and retain only terms linear in these four quantities. We anticipate in this approximation the expression for $\alpha \sim \sqrt{1-\Lambda r_{\mathrm{T}}^{2} / 3} r_{0} / r_{\mathrm{T}}$. We assume that $\lambda:=\sqrt{\Lambda / 3} r_{0} \ll 1$ and only retain terms linear in this and its primed quantity. For the example of the quasar SDSS J1004+4112, all six quantities are approximately $10^{-5}$.

Our aim is to compute the proper time delay $\Delta \tau=$ $\sqrt{B\left(r_{\mathrm{T}}\right)}\left(t_{\mathrm{T}}^{\prime}+t_{\mathrm{S}}^{\prime}-t_{\mathrm{T}}-t_{\mathrm{S}}\right)$ as a function of $M, \alpha, \alpha^{\prime}, r_{\mathrm{T}}, r_{\mathrm{S}}$, and $\Lambda$. 


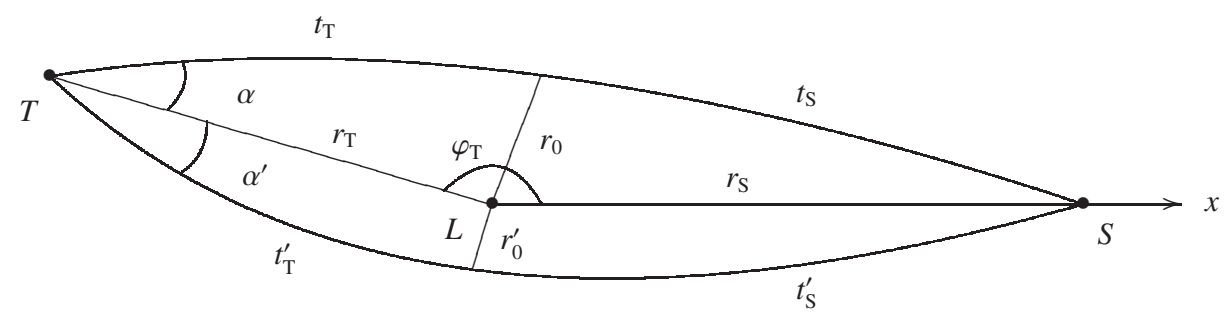

Fig. 1. A double image.

\section{Integrating the geodesics}

We start with the list of the non-vanishing Christoffel symbols for the Kottler metric with $\theta=\pi / 2$ and denote ${ }^{\prime}:=\mathrm{d} / \mathrm{d} r$,

$\Gamma_{t r}^{t}=B^{\prime} /(2 B), \quad \Gamma_{t t}^{r}=B B^{\prime} / 2, \quad \Gamma_{r r}^{r}=-B^{\prime} /(2 B)$,

$\Gamma_{\varphi \varphi}^{r}=-r B, \quad \Gamma_{r \varphi}^{\varphi}=1 / r$.

The geodesic equations read:

$\ddot{t}+B^{\prime} / B \dot{t} \dot{r}=0$,

$\ddot{r}+\frac{1}{2} B B^{\prime} \dot{t}^{2}-\frac{1}{2} B B^{\prime} \dot{r}^{2}-r B \dot{\varphi}^{2}=0$,

$\ddot{\varphi}+2 r^{-1} \dot{r} \dot{\varphi}=0$,

where we denote the affine parameter by $p$ and ${ }^{*}:=\mathrm{d} / \mathrm{d} p$. We then obtain three first integrals:

$$
\begin{aligned}
\dot{t} & =1 / B(r), \\
\dot{\varphi} & =\frac{r_{0}}{r^{2} \sqrt{B\left(r_{0}\right)}}, \\
\dot{r} & =\left(1-\frac{r_{0}^{2}}{r^{2}} \frac{B(r)}{B\left(r_{0}\right)}\right)^{1 / 2} .
\end{aligned}
$$

Eliminating the affine parameter we derive:

$$
\begin{aligned}
& \frac{\mathrm{d} \varphi}{\mathrm{d} r}= \pm \frac{1}{r \sqrt{r^{2} / r_{0}^{2}-1}}\left[1-\frac{2 G M}{r}-\frac{2 G M}{r_{0}} \frac{r}{r+r_{0}}\right]^{-1 / 2}, \\
& \frac{\mathrm{d} t}{\mathrm{~d} r}= \pm \frac{\sqrt{B\left(r_{0}\right)}}{B(r) \sqrt{1-r_{0}^{2} / r^{2}}}\left[1-\frac{2 G M}{r}-\frac{2 G M}{r_{0}} \frac{r}{r+r_{0}}\right]^{-1 / 2} .
\end{aligned}
$$

Integrating Eq. (11), we obtain $\alpha \sim \sqrt{1-\Lambda r_{\mathrm{T}}^{2} / 3} r_{0} / r_{\mathrm{T}}$ and

$$
\frac{r_{\mathrm{T}}}{r_{\mathrm{S}}} \sim \frac{4 G M}{\alpha \alpha^{\prime} r_{\mathrm{T}}}\left(1-\Lambda r_{\mathrm{T}}^{2} / 3\right)-1
$$

As noticed in Schücker (2007), Eq. (13) agrees with Rindler \& Ishak's (2007) result in the aligned case, $\alpha=\alpha^{\prime}$ (to all orders in the cosmological constant).

We now integrate Eq. (12):

$$
\begin{aligned}
t_{\mathrm{T}}= & \sqrt{B\left(r_{0}\right)} \int_{r_{0}}^{r_{\mathrm{T}}} \frac{1}{B(r) \sqrt{1-r_{0}^{2} / r^{2}}} \\
& \times\left[1-\frac{2 G M}{r}-\frac{2 G M}{r_{0}} \frac{r}{r+r_{0}}\right]^{-1 / 2} \mathrm{~d} r \\
\sim & r_{0} \sqrt{B\left(r_{0}\right)}\left[I_{\mathrm{T} 1}+\delta I_{\mathrm{T} 2}+\delta I_{\mathrm{T} 3}+2 \delta I_{\mathrm{T} 4}\right] .
\end{aligned}
$$

We set $y:=r_{0} / r, \epsilon_{\mathrm{T}}:=r_{0} / r_{\mathrm{T}}>\lambda$,

$$
\begin{aligned}
I_{\mathrm{T} 1} & :=\int_{\epsilon_{\mathrm{T}}}^{1} \frac{1}{y^{2}-\lambda^{2}} \frac{\mathrm{d} y}{\sqrt{1-y^{2}}} \\
& =\frac{1}{\lambda \sqrt{1-\lambda^{2}}} \operatorname{arctanh}\left(\frac{\lambda}{\sqrt{1-\lambda^{2}}} \frac{\sqrt{1-\epsilon_{\mathrm{T}}^{2}}}{\epsilon_{\mathrm{T}}}\right),
\end{aligned}
$$

$$
\begin{aligned}
I_{\mathrm{T} 2} & :=\int_{\epsilon_{\mathrm{T}}}^{1} \frac{y}{y^{2}-\lambda^{2}} \frac{\mathrm{d} y}{\sqrt{1-y^{2}}} \\
& =\frac{1}{\sqrt{1-\lambda^{2}}} \operatorname{arctanh} \sqrt{\frac{1-\epsilon_{\mathrm{T}}^{2}}{1-\lambda^{2}}}
\end{aligned}
$$

$$
I_{\mathrm{T} 3}:=\int_{\epsilon_{\mathrm{T}}}^{1} \frac{1}{y^{2}-\lambda^{2}} \frac{1}{1+y} \frac{\mathrm{d} y}{\sqrt{1-y^{2}}}
$$$$
=\frac{1}{2\left(1-\lambda^{2}\right)}\left[2 \frac{\sqrt{1-\epsilon_{\mathrm{T}}^{2}}}{1+\epsilon_{\mathrm{T}}}-\frac{1}{\sqrt{1-\lambda^{2}}}\right.
$$$$
\times \ln \frac{\left(1+\sqrt{1-\lambda^{2}} \sqrt{1-\epsilon_{\mathrm{T}}^{2}}-\lambda \epsilon_{\mathrm{T}}\right)\left(1+\sqrt{1-\lambda^{2}} \sqrt{1-\epsilon_{\mathrm{T}}^{2}}+\lambda \epsilon_{\mathrm{T}}\right)}{\epsilon_{\mathrm{T}}^{2}-\lambda^{2}}
$$$$
\left.-\frac{1}{\lambda \sqrt{1-\lambda^{2}}} \ln \frac{\left(1+\sqrt{1-\lambda^{2}} \sqrt{1-\epsilon_{\mathrm{T}}^{2}}+\lambda \epsilon_{\mathrm{T}}\right)\left(\epsilon_{\mathrm{T}}-\lambda\right)}{\left(1+\sqrt{1-\lambda^{2}} \sqrt{1-\epsilon_{\mathrm{T}}^{2}}-\lambda \epsilon_{\mathrm{T}}\right)\left(\epsilon_{\mathrm{T}}+\lambda\right)}\right]
$$

$$
\begin{aligned}
I_{\mathrm{T} 4}:= & \int_{\epsilon_{\mathrm{T}}}^{1} \frac{y^{3}}{\left(y^{2}-\lambda^{2}\right)^{2}} \frac{\mathrm{d} y}{\sqrt{1-y^{2}}} \\
= & \frac{2-\lambda^{2}}{2 \sqrt{1-\lambda^{2} 3}} \operatorname{arctanh} \sqrt{\frac{1-\epsilon_{\mathrm{T}}^{2}}{1-\lambda^{2}}} \\
& +\frac{\lambda^{2}}{2\left(1-\lambda^{2}\right)} \frac{\sqrt{1-\epsilon_{\mathrm{T}}^{2}}}{\epsilon_{\mathrm{T}}^{2}-\lambda^{2}}
\end{aligned}
$$

Taking $\Lambda$ to be zero, Eq. (14) yields the correct time of flight found in textbooks. The leading term in $\Lambda$ is in the first integral $I_{\mathrm{T} 1} \sim\left[1+\frac{1}{3} \lambda^{2} / \epsilon_{\mathrm{T}}^{2}\right] \epsilon_{\mathrm{T}}+\ldots$ and compares correctly with e.g. 17 of Kagramanova et al. (2006). However, this term cancels inside the expression for the time delay. To compute $t_{\mathrm{T}}^{\prime}-t_{\mathrm{T}}$, we must subtract large numbers that are almost identical and develop the 
relevant differences of the four integrals separately. Setting $x:=$ $\alpha^{\prime} / \alpha \sim r_{0}^{\prime} / r_{0}$ we have:

$$
\begin{aligned}
& \Delta_{\mathrm{T} 1}:=r_{0}^{\prime} \sqrt{B\left(r_{0}^{\prime}\right)} I_{\mathrm{T} 1}^{\prime}-r_{0} \sqrt{B\left(r_{0}\right)} I_{\mathrm{T} 1} \\
& \sim G M\left[-(1 / x-1) \frac{\operatorname{arctanh}\left(\lambda / \epsilon_{\mathrm{T}}\right)}{\lambda}\right. \\
& \left.+\frac{1}{2}\left(1-x^{2}\right) \frac{\epsilon_{\mathrm{T}}}{\delta}-\frac{1}{2}\left(1 / x^{2}-1\right) \delta \frac{\operatorname{arctanh}\left(\lambda / \epsilon_{\mathrm{T}}\right)}{\lambda}\right], \\
& \Delta_{\mathrm{T} 2}:=\sqrt{B\left(r_{0}^{\prime}\right)} I_{\mathrm{T} 2}^{\prime}-\sqrt{B\left(r_{0}\right)} I_{\mathrm{T} 2} \sim \ln x, \\
& \Delta_{\mathrm{T} 3}:=\sqrt{B\left(r_{0}^{\prime}\right)} I_{\mathrm{T} 3}^{\prime}-\sqrt{B\left(r_{0}\right)} I_{\mathrm{T} 3} \\
& \sim(1 / x-1) \frac{\operatorname{arctanh}\left(\lambda / \epsilon_{\mathrm{T}}\right)}{\lambda}+\ln x \\
& -\left(1 / x^{2}-1\right) \delta \frac{\operatorname{arctanh}\left(\lambda / \epsilon_{\mathrm{T}}\right)}{\lambda}, \\
& \Delta_{\mathrm{T} 4}:=\sqrt{B\left(r_{0}^{\prime}\right)} I_{\mathrm{T} 4}^{\prime}-\sqrt{B\left(r_{0}\right)} I_{\mathrm{T} 4} \sim \ln x .
\end{aligned}
$$

The large value of the term $(1 / x-1) \operatorname{arctanh}\left(\lambda / \epsilon_{\mathrm{T}}\right) / \lambda$ cancels when we add the four terms:

$$
\begin{aligned}
t_{\mathrm{T}}^{\prime}-t_{\mathrm{T}} \sim & \Delta_{\mathrm{T} 1}+G M \Delta_{\mathrm{T} 2}+G M \Delta_{\mathrm{T} 3}+2 G M \Delta_{\mathrm{T} 4} \\
\sim & G M\left[\frac{1}{2}\left(1-x^{2}\right) \frac{\epsilon_{\mathrm{T}}}{\delta}\right. \\
& \left.-\frac{3}{2}\left(1 / x^{2}-1\right) \delta \frac{\operatorname{arctanh}\left(\lambda / \epsilon_{\mathrm{T}}\right)}{\lambda}-2 \ln x\right] .
\end{aligned}
$$

Finally, we write the time delay to leading order in $\delta, \epsilon$. and $\lambda$ as,

$$
\begin{aligned}
\Delta \tau \sim & \sqrt{1-\Lambda r_{\mathrm{T}}^{2} / 3} G M\left[\frac{1}{2} \frac{\alpha^{2}-\alpha^{2}}{1-\Lambda r_{\mathrm{T}}^{2} / 3} \frac{r_{\mathrm{T}}}{G M}\left(1+\frac{r_{\mathrm{T}}}{r_{\mathrm{S}}}\right)\right. \\
& -\frac{3}{2}\left(1-\frac{\Lambda}{3} r_{\mathrm{T}}^{2}\right)\left(\frac{1}{\alpha^{\prime 2}}-\frac{1}{\alpha^{2}}\right) \frac{G M}{\sqrt{\Lambda / 3} r_{\mathrm{T}}^{2}} \\
& \left.\times\left(\operatorname{arctanh} \sqrt{\frac{\Lambda}{3}} r_{\mathrm{T}}+\operatorname{arctanh} \sqrt{\frac{\Lambda}{3}} r_{\mathrm{S}}\right)+4 \ln \frac{\alpha}{\alpha^{\prime}}\right] .
\end{aligned}
$$

Our expression for the time delay depends on the cosmological constant for different reasons. The overall factor $\sqrt{1-\Lambda r_{\mathrm{T}}^{2} / 3}$ comes from the proper time correction on Earth and decreases the time delay as the cosmological constant (or the distance between the Earth and the lens) increases. The other $\Lambda$-dependencies are of a geometrical nature. They compete with each other and whether the total time delay is a decreasing or increasing function of $\Lambda$ depends on the particular values of deflection angles and coordinate distances of source and observer from the lens. We note however that the well-known dependence of the physical angular distances on the cosmological constant from the Hubble diagram is not contained in our formula. We remove this dependence from our analysis by computing the angular distances in the next section with a fixed cosmological constant. For the translation from angular distances to coordinate distances, we use one Ansatz independent of $\Lambda$. The competition of the geometrical $\Lambda$-dependencies is illustrated in Table 2 of the next section. We note that for equal deflection angles, $\alpha=\alpha^{\prime}$, the time delay vanishes as expected for symmetry reasons.

Sereno's (2007) Eq. (24) in version 2, provides the time delay only if the source position is known in the absence of the
Table 1. $r_{\mathrm{S}}$ from the first of Eqs. (25).

\begin{tabular}{ccccccccc}
\hline \hline$M \pm 20 \%$ & + & - & - & - & - & + & + & + \\
$\alpha \pm 10 \%$ & + & + & - & - & + & + & - & - \\
$\alpha^{\prime} \pm 10 \%$ & + & + & + & - & - & - & - & + \\
\hline$r_{\mathrm{S}}\left[10^{25} \mathrm{~m}\right]$ & 5.3 & $\mathbf{6 . 1}$ & 5.6 & 5.3 & 5.6 & 5.0 & $\mathbf{4 . 8}$ & 5.0 \\
$\Lambda\left[10^{-52} \mathrm{~m}^{-2}\right]$ & 2.2 & $\mathbf{0 . 7}$ & 1.5 & 2.2 & 1.5 & 2.8 & $\mathbf{3 . 4}$ & 2.8 \\
$\Delta \tau$ [years $]$ & 24.1 & 20.3 & $\mathbf{1 2 . 8}$ & 16.1 & 23.6 & $\mathbf{2 7 . 7}$ & 18.7 & 15.1 \\
\hline
\end{tabular}

lens. Ishak's (2008) recent analysis contains two contributions of the cosmological constant to the time of flight. We have shown that the real difficulty resides however in the computation of the difference between the two times of flight because of the indicated cancelation of large terms. Therefore all contributions must be computed.

\section{SDSS J1004+4112}

Consider the lensing cluster of SDSS J1004+4112 and the quasar as source, Inada et al. (2003), Sharon et al. (2005), Ota et al. (2006). As we have at least 4 images, the cluster cannot be spherically symmetric. We disregard the images with small $\alpha$ and consider only the images $\mathrm{C}$ and $\mathrm{D}$ with $\alpha=10^{\prime \prime} \pm 10 \%$ and $\alpha^{\prime}=5^{\prime \prime} \pm 10 \%$. We assume that these images are less sensitive to a non-spherical inner struture of the cluster. The mass of the cluster is $M=(1 \pm 0.2) \times 10^{44} \mathrm{~kg}$. The cluster has a redshift of $z_{\mathrm{L}}=0.68$. A numerical integration of the Cold Dark Matter model with $\Lambda=1.5 \times 10^{-52} \mathrm{~m}^{-2}$ yields an area distance $d_{\mathrm{L}}=7.0 \times 10^{25} \mathrm{~m}$ as seen from Earth. This area distance coincides with the coordinate distance $r_{\mathrm{T}}$. For the quasar, we have $z_{\mathrm{S}}=1.734$ yielding the area distance $d_{\mathrm{S}}=13.7 \times 10^{25} \mathrm{~m}$. Due to the magnification of the quasar by the cluster, the translation from its area distance to its coordinate distance is ambiguous: it depends on which image is used and in the case of alignment of source, lens, and observer, the area distance can even be zero. Therefore we use the area distance in absence of the lens, $M=0$. Then remain two possibilities, $\Lambda \neq 0$ and $\Lambda=0$. We use both Ansätze,

$d_{\mathrm{S}}=\frac{r_{\mathrm{T}}+r_{\mathrm{S}}}{\sqrt{1-\Lambda r_{\mathrm{S}}^{2} / 3}}$ or $\quad d_{\mathrm{S}}=r_{\mathrm{T}}+r_{\mathrm{S}}$

The first expression gives $r_{\mathrm{S}}=5.3 \times 10^{25} \mathrm{~m}, \Lambda=2.2 \times 10^{-52} \mathrm{~m}^{-2}$ from Eq. (13) and $\Delta \tau=20.1$ years. The second gives $r_{\mathrm{S}}=6.7 \times$ $10^{25} \mathrm{~m}, \Lambda=2.7 \times 10^{-52} \mathrm{~m}^{-2}$ and $\Delta \tau=17.1$ years. The errors in these quantities arise from errors in $M, \alpha$, and $\alpha^{\prime}$, and can be read from Tables 1 and 2. Table 1 summarizes the values of $r_{\mathrm{S}}$ for the first of Eqs. (25), $\Lambda$, and $\Delta \tau$ at the corners of the error box in $M, \alpha$ and $\alpha^{\prime}$. Table 2 summarizes the values of $\Lambda$ and $\Delta \tau$ for $r_{\mathrm{S}}=d_{\mathrm{S}}-r_{\mathrm{T}}=6.7 \times 10^{25} \mathrm{~m}$ at the corners of the error box. Minimal and maximal values are are indicated in bold face from which we obtain:

$$
\begin{aligned}
& \Lambda=(2.0 \pm 1.4) \times 10^{-52} \mathrm{~m}^{-2} \text { or }(2.4 \pm 1.5) \times 10^{-52} \mathrm{~m}^{-2} \\
& \Delta \tau=(20.2 \pm 7.5) \text { years } \text { or } \quad(18.1 \pm 6.1) \text { years }
\end{aligned}
$$

It is encouraging to note that both Ansätze yield similar results both for the cosmological constant and the time delay, and that both constraints shown in Eq. (26) on the cosmological constant are compatible with the present observational bounds, $\Lambda=(1.36 \pm 0.3) \times 10^{-52} \mathrm{~m}^{-2}$, whose central value we have used, without variation, to translate redshifts into area distances. Both constraints shown in Eq. (27) on the time delay are compatible 
Table 2. $r_{\mathrm{S}}=d_{\mathrm{S}}-r_{\mathrm{T}}=6.7 \times 10^{25} \mathrm{~m}$.

\begin{tabular}{ccccccccc}
\hline \hline$M \pm 20 \%$ & + & - & - & - & - & + & + & + \\
$\alpha \pm 10 \%$ & + & + & - & - & + & + & - & - \\
$\alpha^{\prime} \pm 10 \%$ & + & + & + & - & - & - & - & + \\
\hline$\Lambda\left[10^{-52} \mathrm{~m}^{-2}\right]$ & 2.7 & $\mathbf{0 . 9}$ & 1.9 & 2.7 & 1.9 & 2.3 & $\mathbf{3 . 9}$ & 3.3 \\
$\Delta \tau[$ years $]$ & 21.8 & 19.6 & $\mathbf{1 2 . 0}$ & 14.6 & 22.0 & $\mathbf{2 4 . 2}$ & 16.0 & 13.3 \\
\hline
\end{tabular}

with the observational lower bound of $\Delta \tau>5.7$ years measured by Fohlmeister et al. (2007) $\Delta \tau>5.7$ years.

\section{Conclusions}

Our computation is based on three tentative assumptions:

- The sphericity of the cluster.

- The velocity of observer and source with respect to the lens is negligible.

- All masses apart that of the cluster are negligible.

The first assumption raises an old question about whether a spherical cow can be useful. To go beyond this assumption requires consideration of multiple scattering of the photon off subconstituents of the cluster. Calculating angles, time delays, and the number of images from the density profile of the cluster and its dark matter halo is a well developed art, e.g. Schneider et al. (1992). Applying the method to SDSS J1004+4112, Kawano \& Oguri (2006) predict a time delay between images C and D of up to 10 years. However including a positive cosmological constant in their analysis is not straightforward.

Khriplovich \& Pomeransky (2008) point out that if the Earth is taken comoving with respect to the exponentially expanding de Sitter space then the effect of the cosmological constant on the deflection cancels.

Going beyond the third idealisation necessitates an interpolating solution, which embeds many static, curved Kottler solutions into the ambient expanding, flat Friedmann solution. This is a long standing problem, to which Einstein \& Straus (1946) and Schücking (1954) have contributed an unstable solution, see Krasiński (1997). A first qualitative assessment based on this solution is given in Sect. 3 of Ishak et al. (2007): the other clusters in the universe weaken the effect of the cosmological constant and increase upper bounds on the cosmological constant from certain lenses by two orders of magnitude.

We conclude that we agree with previous authors that lensing and time delay on a cosmological scale is a subject certainly worthy of study and that our theoretical understanding of it is incomplete. For the older physicists among us however, a time delay of 10 or 20 years is bad news. By the time the expected signal in image $\mathrm{D}$ arrives, we might already be gone.

Note added in proof Ishak (2008) has extended the analysis of how the embedding of Kottler's solution into Friedmann's solution modifies light deflection to include the time of flight. We thank him for having kindly sent us his work.

\section{References}

Bakala, P., et al. 2007 [arXiv: 0709 . 4274]

Einstein, A., \& Straus, E. G. 1946, Rev. Mod. Phys., 17, 120, and 18, 148 Fohlmeister, J., et al. 2007 [arXiv: 0710.1634]

Inada, N., Oguri, M., Pindor, B., et al. 2003, Nature, 426, 810

Oguri, M., Inada, N., Keeton, C. R., et al. 2004, ApJ, 605, 78

Ishak, M. 2008 [arXiv: 0801.3514]

Ishak, M., et al. 2007 [arXiv: 0710.4726]

Kagramanova, V., Kunz, J., \& Lämmerzahl, C. 2006, Phys. Lett. B, 634, 465

Kawano, Y., \& Oguri, M. 2006, Publ. Astron. Soc. Jap., 58, 271

Kerr, A. W., Hauck, J. C., Mashhoon, B., et al. 2003, Class. Quant. Grav., 20, 2727

Khriplovich, I. B., \& Pomeransky, A. A. 2008 [arXiv: 0801. 1764]

Krasiński, A. 1997, Inhomogeneous Cosmological Models (Cambridge University Press), 113

Lake, K. 2007 [arXiv: 0711.0673]

Ota, N., Inada, N., Oguri, M., et al. 2006, ApJ, 647, 215

Rindler, W., \& Ishak, M. 2007, Phys. Rev. D76, 043006

Schneider, P., Ehlers, J., \& Falco, E. 1992, Gravitational Lenses, Springer

Schücker, T. 2007, Gen. Rel. Grav., in press [arXiv: 0712 . 1559]

Schücking, E. 1954, Z. Phys., 137, 595

Sereno, M. 2007 [arXiv:0711.1802]

Sharon, K., Ofek, E. O., Smith, G. P., et al. 2005, ApJ, 629, L73 